\title{
More strata and another GATA: Novel roles for GATA4 in postinfarct myocardial repair
}

\author{
Leora B. Balsam, MD
}

\author{
From the Department of Cardiothoracic Surgery, New York University-Langone Medical Center, New York, NY. \\ Disclosures: Author has nothing to disclose with regard to commercial support. \\ Received for publication July 4, 2017; accepted for publication July 10, 2017; available ahead of print Aug 5, \\ 2017. \\ Address for reprints: Leora B. Balsam, MD, Department of Cardiothoracic Surgery, NYU-Langone Medical Cen- \\ ter, 530 First Ave, Suite 9V, New York, NY 10016 (E-mail: leora.balsam@nyumc.org). \\ J Thorac Cardiovasc Surg 2017;154:1611-2 \\ 0022-5223/\$36.00 \\ Copyright (C) 2017 by The American Association for Thoracic Surgery \\ http://dx.doi.org/10.1016/j.jtcvs.2017.07.002
}

Just when you think dogma has become truth, the science of regenerative medicine opens a new frontier. We have known for years that the adult human heart has limited regenerative capacity and its primary response to injury is fibrosis. In the context of myocardial infarction, this fibrotic response results in adverse remodeling, characterized by left ventricular dilatation, secondary mitral regurgitation, and worsening ventricular function with time. At the turn of the century, the focus was on cell-based therapies after infarction, with a shift from embryonic stem cells, which were no longer readily available because of regulatory policy, to adult bone marrow-derived stem cells. Major problems with cell-based therapies included limited (if any) ability to transdifferentiate into functional cardiomyocytes, limited survival in the infarct milieu, and a time delay relative to acute myocardial infarction for preparation of autologous cells for transplantation. Although cell-based therapies have been used in clinical trials, results have been disappointing, leading scientists to explore alternate therapies.

A radically different approach, direct cellular reprogramming, has emerged as a promising methodology for myocardial regeneration. ${ }^{1,2}$ The premise is that cardiac fibroblasts, which along with endothelial cells comprise $60 \%$ of the heart's cells, can be coaxed into a cardiomyocyte phenotype with a cocktail of reprogramming factors. These factors include cardiogenic transcription factors GATA4, Mef2c, and Tbx5, which can be experimentally overexpressed in cardiac fibroblasts both in vitro and in vivo by means of viral vectors. In vitro, the reprogramming efficiency of this 3transcription factor cocktail, known as GMT, is higher in neonatal cardiac fibroblasts than in adult cardiac fibroblasts, and the resulting reprogrammed cells are known as induced cardiomyocyte-like cells. These cells express cardiomyocyte-specific proteins, and although many have features of immature cardiomyocytes, a minority beat spontaneously and have electrophysiologic characteristics of adult cardiomyocytes. In vivo, local delivery of GMT to the infarct region results in markedly less fibrosis

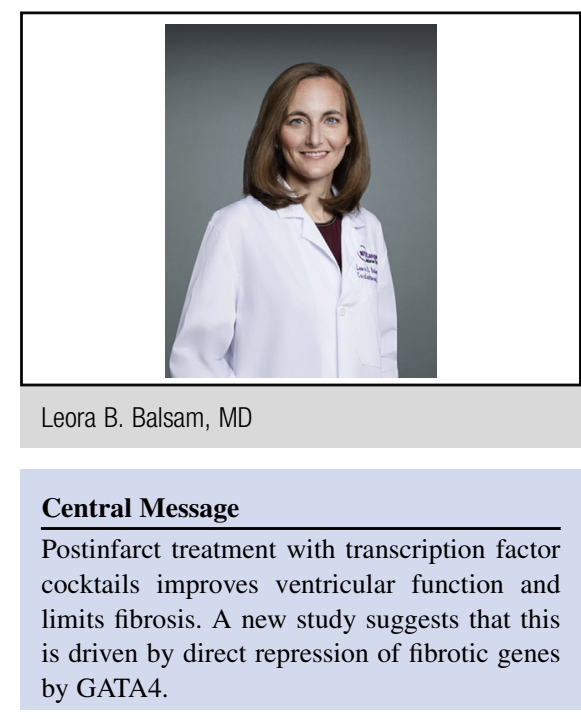

See Article page 1601.

and improved left ventricular function. Although investigators have shown that GMT treatment in the infarct model results in direct cellular reprogramming of a small subset of cardiac fibroblasts to induced cardiomyocyte-like cells, ${ }^{2}$ the degree of change in fibrosis and ventricular function with time has seemed out of proportion to the relative inefficiency of cellular reprogramming. Could another mechanism, other than direct cellular reprogramming, explain these marked changes in structure and function?

In this issue of the Journal, Mathison and colleagues ${ }^{3}$ hypothesized that GMT (or its components) has direct effects on fibrotic signaling, independent of cellular reprogramming. They report that GATA4 (the $G$ of $G M T$ ) is the key player that directly represses a signature of fibrotic genes, including $\alpha$-1 type 1 collagen, connective tissue growth factor, fibronectin, and Snail. In vitro, treatment of rat cardiac fibroblasts with GATA4 lentiviral vector alone (as well as GMT triple vectors) results in a shift from epithelialmesenchymal transition toward mesenchymal-epithelial transition. Because epithelial-mesenchymal transition is a driving process in postinfarct fibrosis and remodeling, favoring the balance of proliferative myofibroblasts over quiescent fibroblasts, strategies that abrogate this process may have clinical utility. The transcription factor Snail is a known regulator of epithelial-mesenchymal transition, and Mathison and colleagues ${ }^{3}$ show that GATA4 influences Snail's transcription through direct interaction with histone 
deacetylase 2 at the Snail promoter. These findings complement those of Muraoka and colleagues, ${ }^{4}$ who found that knockdown of Snail in fibroblasts treated with GMT results in suppression of fibroblast genes and upregulation of cardiac gene expression.

Mathison and colleagues ${ }^{3}$ also report that in a rat left anterior descending coronary artery ligation model, treatment of the infarct zone with lentivirus encoding GATA4 alone results in significantly less fibrosis 4 weeks after infarction relative to control animals. Moreover, both GATA4 alone and GMT triple vector treatment significantly improve postinfarct left ventricular function relative to control animals.

These authors are no strangers to the science of postinfarct myocardial repair and direct cellular reprogramming. ${ }^{5-7}$ Their previous work includes investigation of the efficacy of nonintegrating viruses for GMT delivery, evaluation of lentiviral polycistronic vectors for GMT delivery, and assessment of scar prevascularization for direct cellular reprogramming with GMT. What differs in the study in this issue ${ }^{3}$ is the focus on the role of GMT (and its components) on fibrosis rather than myocardial regeneration. Previous reports linking GATA4 to the fibrotic process are few, so these mechanistic experiments offer a novel viewpoint on how treatment with cardiac reprogramming cocktails that include GATA4 may limit adverse postinfarct remodeling.

GATA4 is best known for its role in cardiac development, in which loss of function mutations are associated with congenital defects in septation and valve structure. ${ }^{8}$ Although GATA4's function in the postnatal heart is less defined, it may promote compensatory cardiomyocyte hypertrophy. ${ }^{9}$ The study of Mathison and colleagues ${ }^{3}$ also lends evidence for a regulatory role of GATA4 in compensatory myocyte hypertrophy, but more importantly, it proposes a role in repressing myocardial fibrosis. Interestingly, Bian and associates ${ }^{10}$ had previously shown that GATA4 overexpression in cardiac fibroblasts used for cell therapy after infarction also helped promote beneficial ventricular remodeling and improved left ventricular function.

For the cardiac surgeon, how do these experiments intersect with clinical practice? Many obstacles remain before
GATA4 modulation can be harnessed to impede myocardial fibrosis. Lentiviral gene delivery systems, which integrate into proliferating and nonproliferating cells, are not an option because of the potential for insertional mutagenesis. Mathison and coworkers ${ }^{5}$ have explored the use of nonintegrating adenoviral vectors; however, these vectors have their limitations, including transient expression and activation of an immune response. Because GATA4 functions intracellularly, modulation of its activity can only occur at a transcriptional or translational level. What remains clear is that this experimental science is just that, with a multitude of barriers to be overcome before clinical implementation.

\section{References}

1. Ieda M, Fu JD, Delgado-Olguin P, Vedantham V, Hayashi Y, Bruneau BG, et al. Direct reprogramming of fibroblasts into functional cardiomyocytes by defined factors. Cell. 2010;142:375-86.

2. Song K, Nam YJ, Luo X, Qi X, Tan W, Huang GN, et al. Heart repair by reprogramming non-myocytes with cardiac transcription factors. Nature. 2012;485: 599-604.

3. Mathison M, Singh VP, Sanagasetti D, Yang L, Pinnamaneni JP, Yang J, et al. Cardiac reprogramming factor Gata4 reduces post-infarct cardiac fibrosis through direct repression of the profibrotic mediator snail. J Thorac Cardiovasc Surg. 2017;154:1601-10.e3.

4. Muraoka N, Yamakaw H, Miyamoto K, Sadahiro T, Umei T, Isomi M, et al. MiR133 promotes cardiac reprogramming by directly repressing Snail and silencing fibroblast signatures. EMBO J. 2014;33:1565-81.

5. Mathison M, Singh VP, Chiuchiolo MJ, Sanagasetti D, Mao Y, Patel VB, et al. In situ reprogramming to transdifferentiate fibroblasts into cardiomyocytes using adenoviral vectors: implications for clinical myocardial regeneration. J Thorac Cardiovasc Surg. 2017;153:329-39.e3.

6. Mathison M, Singh VP, Gersch RP, Ramirez MO, Cooney A, Kaminsky SM, et al. "Triplet" polycistronic vectors encoding Gata4, Mef2c, and Tbx5 enhances postinfarct ventricular functional improvement compared with singlet vectors. J Thorac Cardiovasc Surg. 2014;148:1656-64.e2.

7. Mathison M, Gersch RP, Nasser A, Lilo S, Korman M, Fourman M, et al. In vivo cardiac cellular reprogramming efficacy is enhanced by angiogenic preconditioning of the infarcted myocardium with vascular endothelial growth factor. J Am Heart Assoc. 2012;1:e05652.

8. Moskowitz IP, Wang J, Peterson MA, Pu WT, Mackinnon AC, Oxburgh L, et al. Transcription factor genes Smad4 and Gata4 cooperatively regulate cardiac valve development [corrected]. Proc Natl Acad Sci U S A. 2011;108: 4006-11.

9. Bisping E, Ikeda S, Kong SW, Tarnavski O, Bodyak N, McMullen JR, et al. Gata4 is required for maintenance of postnatal cardiac function and protection from pressure overload-induced heart failure. Proc Natl Acad Sci U S A. 2006;103: 14471-6.

10. Bian J, Popovic ZB, Benejam C, Kiedrowski M, Rodriguez LL, Penn MS. Effect of cell-based intercellular delivery of transcription factor GATA4 on ischemic cardiomyopathy. Circ Res. 2007;100:1626-33. 